OPEN ACCESS

Edited by:

Jinwen Shi,

Xi'an Jiaotong University, China

Reviewed by:

Xiaohui Liu,

Tianjin Polytechnic University, China Paweł Chmielarz,

Rzeszów University of Technology, Poland

*Correspondence:

Christian Rolando

christian.rolando@univ-lille.fr

Specialty section:

This article was submitted to Organic Chemistry,

a section of the journal

Frontiers in Chemistry

Received: 01 April 2020

Accepted: 17 July 2020 Published: 08 September 2020

Citation:

El Achi N, Bakkour Y, Adhami W, Molina J, Penhoat M, Azaroual N, Chausset-Boissarie $L$ and Rolando $C$ (2020) Metal-Free ATRP Catalyzed by

Visible Light in Continuous Flow.

Front. Chem. 8:740.

doi: $10.3389 /$ fchem.2020.00740

\section{Metal-Free ATRP Catalyzed by Visible Light in Continuous Flow}

\author{
Nassim El Achi ${ }^{1}$, Youssef Bakkour ${ }^{2}$, Wissal Adhami ${ }^{1,2}$, Julien Molina ${ }^{1}$, Maël Penhoat ${ }^{1}$, \\ Nathalie Azaroual ${ }^{3}$, Laëtitia Chausset-Boissarie ${ }^{1}$ and Christian Rolando ${ }^{1 *}$ \\ ${ }^{1}$ MSAP 'Miniaturisation pour la Synthèse l'Analyse et la Protéomique', Université de Lille, USR CNRS 3290, Lille, France, \\ 2 Laboratory of Applied Chemistry, Faculty of Sciences III, Lebanese University, Tripoli, Lebanon, ${ }^{3}$ Laboratoire de Physique et \\ d'Application RMN, GRITA 'Groupe de Recherche sur les formes Injectables et les Technologies Associées', Université de \\ Lille, EA 7365, Lille, France
}

ATRP of methyl methacrylate catalyzed by Eosin Y, an inexpensive and an environmental benign dye, was performed in a continuous flow reactor made of FEP tubing and irradiated by visible light green LEDs. The reaction under flow conditions was significantly more rapid and controlled compared to that in batch giving 90\% of polymerization after only $3 \mathrm{~h}$ of irradiation. The formed polymers in flow have $M_{n}$ measured by GPC and DOSY NMR in accordance with the theoretical values and show low dispersities $(\boxplus<1.5)$. The livingness of the polymers has been confirmed by LED on and LED off experiments and by the synthesis of block copolymers. The protocol described herein serves as a "proof of concept" of using Eosin $Y$ as a photocatalyst for controlled polymerization and of using 1D and 2D NMR for polymer characterization. The protocol could be replicated in the future for other reversible-deactivation radical polymerizations.

\section{Keywords: ATRP, continuous flow, visible light, Eosin Y, DOSY NMR}

\section{INTRODUCTION}

During the last decade, much attention has been given to reversible deactivation radical polymerization (RDRP) in polymer and material chemistry, as it is considered a reliable technique for the production of controlled/living polymers offering a wide range of methods like nitroxidemediated radical polymerization (NMP), reversible addition-fragmentation chain transfer (RAFT), variations of organometallic-mediated polymerizations (OMBP), and atom transfer radical polymerization (ATRP) (Gao and Matyjaszewski, 2006; Chmielarz et al., 2017; Fantin et al., 2017).

ATRP has become widely employed due to its remarkable performance in the synthesis of polymers of well-defined chemical composition and complex architecture (Matyjaszewski and Xia, 2001; Ouchi et al., 2008, 2009; Matyjaszewski and Tsarevsky, 2009, 2014; Matyjaszewski, 2012; Boyer et al., 2016; Ribelli et al., 2019).

In an aim to develop more sustainable and visible light-mediated ATRP (Corrigan et al., 2019), researchers have developed photoredox catalytic systems using catalysts like copper $(\mathrm{Cu})$ (Tasdelen et al., 2011; Konkolewicz et al., 2012; Mosnacek and Ilcikova, 2012; Anastasaki et al., 2014; Ribelli et al., 2014; Yang et al., 2015), cyclometallated iridium (Ir) (Fors and Hawker, 2012; Ma et al., 2014, 2015; Treat et al., 2014a; Xu et al., 2014) or ruthenium (Ru) (Zhang et al., 2011; Alfredo et al., 2012; Priyadarshani et al., 2013).

Despite their effectiveness, metal contamination limits their interest for biomedical and electronic applications. Significant effort has therefore been dedicated to develop photoredox metal-free ATRP (Chen et al., 2016; Pan et al., 2016b; Hu et al., 2017; Ryan et al., 2017; Discekici et al., 2018). Pioneering work by Hawker's group demonstrated that 10-phenylphenothiazine 
(PTH) can be an effective organic photoredox catalyst for the polymerization of methacrylates under ultraviolet irradiation (Treat et al., 2014b). Subsequently, the group of Matyjaszewski has extended the methodology to polyacrylonitrile synthesis (Pan et al., 2015).

More importantly, a photoredox ATRP mediated by visible light using organic photoredox catalysts like perylene and fluorescein has been reported with the latter requiring prolonged irradiation times (Miyake and Theriot, 2014; Liu et al., 2016). However, some drawbacks are still present like low initiator efficiency that leads to polymers of elevated masses and dispersity $(\boxplus)$.

To address this challenge (Discekici et al., 2018), the group of Miyake introduced diphenyl dihydrophenazine derivatives (Theriot et al., 2016; Ryan et al., 2017) and recently dimethyl dihydroacridines as metal-free photocatalysts (Buss et al., 2020). Yagci's group also recently reported a metal-free photoinduced ATRP using Eosin Y (Figure 1, Figure S2) (Kutahya et al., 2016; Yilmaz and Yagci, 2018). Their work gave promising results but with some challenges regarding dispersity and reaction time. To overcome these issues, a continuous photoflow reactor can be used since the short optical lengths of flow microreactors can significantly improve sample irradiation and heat and mass transfer that allow the use of more concentrated photoinitiators (Table S1, Figure S3) (Elliott et al., 2014; Garlets et al., 2014; Su et al., 2014; Cambié et al., 2016). Consequently, flow chemistry has recently been used in polymer synthesis since it provides improved initiator efficiency and better-controlled polymers of narrow dispersity compared to classical batch processes (Tonhauser et al., 2012; Chatani et al., 2014; Myers et al., 2014; Reis et al., 2020). However, this field is not widely studied with only a few articles addressing ATRP in flow (Wenn et al., 2014; Melker et al., 2015; Hu et al., 2016; Corrigan et al., 2017; Ramakers et al., 2017; Rubens et al., 2017; Zhang et al., 2019), including those using non-commercially available photoredox catalysts (Ramsey et al., 2017; Ryan et al., 2017; Buss and Miyake, 2018).

Since our research group focuses on performing a wide range of photocatalytic reactions in flow, we herein present the use of Eosin $\mathrm{Y}$ as a photocatalyst for ATRP in flow using visible light. The study falls in the category of "proof of concept" where it aims to determine whether using this cheap and commercially available photocatalyst for ATRP in flow would provide better results than those obtained in batch by Yagci group (Kutahya et al., 2016; Yilmaz and Yagci, 2018) and therefore can be used for other reversible-deactivation radical polymerizations.

\section{MATERIALS}

\section{Chemicals}

Tris(2,2'-bipyridyl)ruthenium(II) chloride hexahydrate $\left(\mathrm{Ru}(\text { bpy })_{3} \mathrm{Cl}_{2} \cdot 6 \mathrm{H}_{2} \mathrm{O}\right.$, 99.95\%) was purchased from Strem Chemicals Inc. (Newbury Port, MA, USA); Eosin Y was purchased from Alfa Aesar (Haverhill, MA, USA); and copper(II) bromide $\left(\mathrm{CuBr}_{2}, 99 \%\right)$ and all other reagents were purchased from Sigma-Aldrich (St. Louis, MO, USA). All solvents were

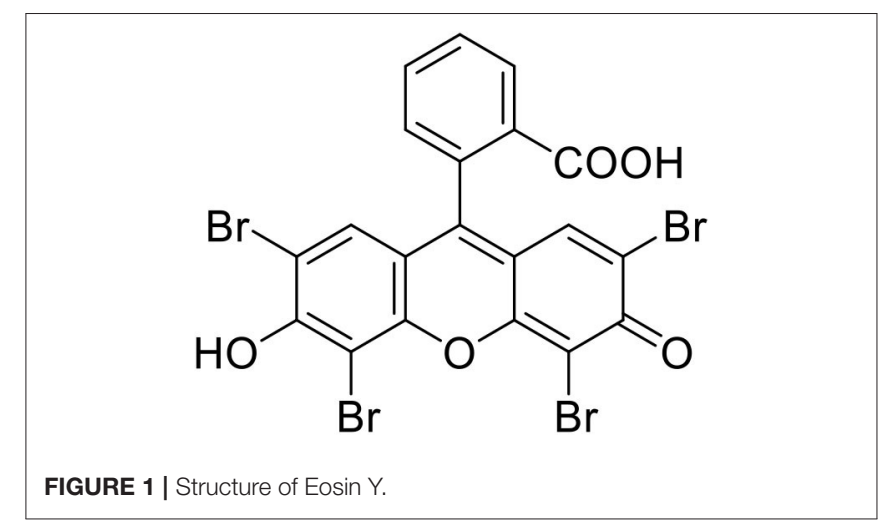

purchased dry from Sigma-Aldrich (St. Louis, MO, USA) and used as received unless otherwise stated.

Anhydrous $N, N$-diisopropylethylamine ( $i$ - $\operatorname{Pr}_{2} \mathrm{NEt}, \geq 99 \%$ ) was further distilled over $\mathrm{KOH}$ and stored in the dark under argon before usage. Methyl methacrylate (99\%) was passed over alumina to remove the hydroquinone stabilizer immediately prior to use.

\section{Flow System}

The lab-designed microreactor is composed of FEP (fluorinated ethylene propylene) tubing (i.d. $800 \mu \mathrm{m}$, length $1.20 \mathrm{~m}$, volume $\approx 2.4 \mathrm{~mL}$, Cluzeau Info Labo (C.I.L.), Sainte-Foy-La-Grande, France) (Figure S1). Two of these reactors were prepared to fit the UV and the Visible LED irradiation systems. For UV irradiation, the tubing was fitted on a metallic grid to allow heat evacuation.

\section{Syringe Pump}

The different flow rates of the reactions performed were regulated using a Harvard Apparatus (Holliston, MA, USA) PHD ULTRA XF syringe pump fitted with 8-mL stainless-steel syringes (THREAD 1/4-28 inch, $\varnothing=1 / 16$ inch, PC5 702267, Harvard Apparatus).

\section{LED Systems}

Blue $(\lambda=450 \mathrm{~nm})$ and green $(\lambda=530 \mathrm{~nm})$ high-power LEDs ( $50 \mathrm{~W}$ electrical power, 4500 lumen, $0.02 \mathrm{~W} \cdot \mathrm{cm}^{-2}$ ) from Bridgelux (Livermore, CA, USA) were used for the photoinduced ATRP catalyzed by $\mathrm{Ru}(\mathrm{bpy})_{3} \mathrm{Cl}_{2}$ and Eosin $\mathrm{Y}$, respectively. OmniCure ${ }^{\circledR}$ AC7300 LEDs of $\lambda=365 \mathrm{~nm}$ (irradiance up to $3 \mathrm{~W} \cdot \mathrm{cm}^{-2}$ ) purchased from Lumen Dynamics (Mississauga, Canada) were used for the photoinduced ATRP catalyzed by $\mathrm{CuBr}_{2}$.

\section{METHODS}

\section{General Procedure of ATRP in Batch}

A Schlenk tube charged with DMF ( $2 \mathrm{~mL}, 50 \%$ v/v vs. monomer), photocatalyst $(2 \mu \mathrm{mol})$, and TPMA or PMDETA ligand for copper-catalyzed ATRP was sealed with rubber septum and purged with nitrogen during $20 \mathrm{~min}$. Methyl methacrylate $(2 \mathrm{~mL}$, $18.8 \mathrm{mmol})$, which was passed over alumina before use, EBiB (17 


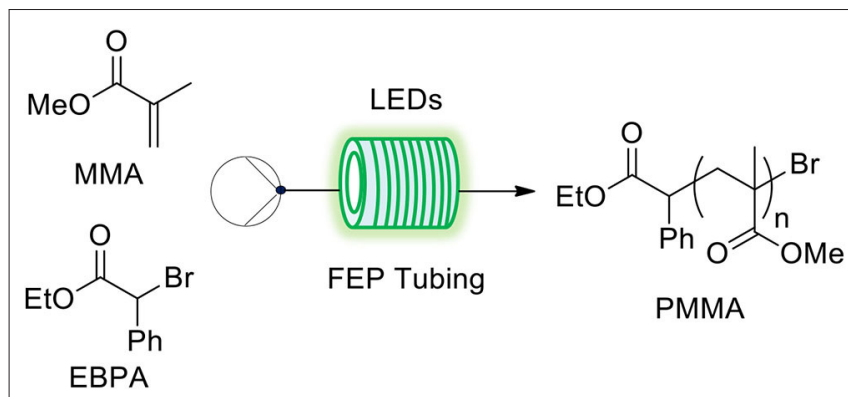

Catalyst

FIGURE 2 | Polymerization systems studied in flow.

$\mu \mathrm{L}, 0.1 \mathrm{mmol})$, and freshly distilled $i$-Pr 2 NEt $(170 \mu \mathrm{L}, 1 \mathrm{mmol})$ were then added under nitrogen. The reaction mixture was irradiated with LEDs. UV LEDs $\left(200 \mathrm{~mW} \cdot \mathrm{cm}^{-2}, 365 \mathrm{~nm}\right)$ were used for the $\mathrm{CuBr}_{2}$ /Ligand catalytic system; blue LEDs ( $50 \mathrm{~W}$, 4500 lumens, $450 \mathrm{~nm}$ ) for $\mathrm{Ru}(\mathrm{bpy}){ }_{3} \mathrm{Cl}_{2}$ and green LEDs ( $50 \mathrm{~W}$, 4500 lumens, $530 \mathrm{~nm}$ ) for Eosin Y. An aliquot was analyzed by ${ }^{1} \mathrm{H}$ NMR to determine the \% of polymerization and by DOSY NMR to determine its $M_{w}$. The formed polymer was then precipitated in methanol, filtered, and dried under vacuum overnight. A solution of $6 \mathrm{mg} / \mathrm{mL}$ in THF was prepared from the dry polymer to be analyzed by GPC.

\section{General Procedure for ATRP in Flow}

ATRP using the three differ rent catalytic systems with the same composition were performed under flow conditions (Figure 2, Figure S4). The degassed reaction mixture was transferred into a lab-designed FEP tubing reactor (i.d. $800 \mu \mathrm{m}$ ) that was illuminated by LEDs of specific wavelengths depending on the catalytic system (Figure S1).

\section{ATRP Using Eosin Y Catalyst Illuminated by Green LEDs in Flow}

Using the same composition as that in the batch conditions, [MMA]: [initiator]: [Eosin Y]: $\left[i-\mathrm{Pr}_{2} \mathrm{NEt}\right]=200: 1: 0.02: 10$ and MMA: $D M F=1: 1(\mathrm{v} / \mathrm{v}), 4 \mathrm{~mL}$ of the reaction mixture was injected within the lab-designed microfluidic reactor (FEP tubing, i.d. $800 \mu \mathrm{m}$ ) that was placed in direct contact with the green LEDs. The irradiation time was varied depending on the flow rate that was adjusted by the syringe pump. For each of the two initiators, EBPA and EBiB, six points that correspond to six different irradiation times were performed. For each point, the $\%$ of conversion was determined by ${ }^{1} \mathrm{H}$ NMR and the polymers were precipitated, filtered, dried, and stored in THF for further analysis by GPC. Note that working under oxygen-free conditions was insured, even during the transfer of the reaction mixture from the Schlenk tube to the syringe. The variation of the \% of conversion of each polymer vs. the irradiation time was used to plot the kinetic curves of ATRP in flow.

\section{Polymer Characterization GPC Analysis}

Size exclusion chromatography (SEC) was performed at room temperature using a Viscotek GPC max system equipped with a Viscotek guard column $(10 \times 4.6 \mathrm{~mm})$ and two Viscotek columns LT 5000-L mixed medium $(300 \times 7.8 \mathrm{~mm})$ fitted with a Viscotek VE 3580 refractometric detector and a Viscotek VE $3210 \mathrm{UV} / \mathrm{Vis}$ detector. THF was used as solvent with a flow rate of $1 \mathrm{~mL} \cdot \mathrm{min}^{-1}$. All molecular weights $\left(M_{n}\right)$ and molecular weight distributions (dispersity, $M_{w} / M_{n}, D$ ) were determined by calibration to known, standard poly(methyl methacrylate) samples purchased from Polymer Laboratories (Church Stretton, United Kingdom).

\section{NMR Analysis}

Proton and carbon magnetic resonance spectra $\left({ }^{1} \mathrm{H}\right.$ NMR and ${ }^{13} \mathrm{C}$ NMR) were recorded on a Bruker AVANCE 300 spectrometer $\left({ }^{1} \mathrm{H} 300 \mathrm{MHz}\right.$ and $\left.{ }^{13} \mathrm{C} 75 \mathrm{MHz}\right)$ using tetramethylsilane (TMS) as the internal standard. Chemical shifts, $\delta$, are given in ppm and coupling constants, $J$, in $\mathrm{Hz} .{ }^{1} \mathrm{H}$ NMR data are reported as follows: chemical shift, multiplicity ( $\mathrm{s}=$ singlet, $\mathrm{d}=$ doublet, $\mathrm{t}=$ triplet, $\mathrm{dd}=$ doublet of doublets, $\mathrm{dt}=$ doublet of triplets, $\mathrm{td}=$ triplet of doublets, $\mathrm{m}=$ multiplet, brs = broad singlet), coupling constants, and integration (Figure S5).

\section{DOSY Analysis}

DOSY experiments were performed on a Bruker AVANCE 500 spectrometer equipped with an ATMA TXI probe with a $\mathrm{z}$ axis gradient coil. All experiments were run without spinning to avoid convection at temperature of $295 \mathrm{~K}$. Maximum gradient strength was $5.35 \mathrm{G} \cdot \mathrm{mm}^{-1}$, and the gradient strengths were varied from 1 to $35 \mathrm{G} \cdot \mathrm{cm}^{-1}$. The standard Bruker pulse program, dstebpgp3s, employing double-stimulated echo sequence and 3spoil gradient, was utilized. Bipolar gradients were used with a total duration of $4 \mathrm{~ms}$. Gradient recovery delay was $100 \mu \mathrm{s}$, diffusion time was $200 \mathrm{~ms}$, and the number of gradient steps was set to be 32. Diffusion coefficients were calculated from a T1/T2 analysis module of Topspin 2.1.

Similar to GPC, 2D-NMR is reported to be used for the determination of the weight average molecular mass $M_{w}$ of the polymer. We run DOSY NMR for PMMA standards of known $M_{w}$ at $20^{\circ} \mathrm{C}$ in $\mathrm{CDCl}_{3}$ instead of benzene- $d^{6}$ according to the protocol described by $\mathrm{Li}$ and coworkers (Alfredo et al., 2012). Each standard gave a value of diffusion coefficient $(\nexists)$ in $\mathrm{m}^{2} \cdot \mathrm{s}^{-1}$ whose logarithmic value was plotted as function of the logarithmic value of the corresponding $M_{w}$. The calibration curve represented in Figure 3 was obtained. The plot has an excellent linearity between $\log D$ vs. $\log M_{w}$ with $R^{2}=0.999$ showing the efficiency of the used protocol. Seven samples of PMMA polymers chosen randomly whose $M_{w}$ values were determined by GPC were analyzed by DOSY to determine their diffusion coefficients using the same protocol of that of the standards $\left(\mathrm{CDCl}_{3}, 20^{\circ} \mathrm{C}\right)$. Knowing the value of $D$ and by using the equation of the calibration curve $\log D=-0.4656 \times \log M_{w}-7.9116$, the $M_{w}$ values of synthesized PMMA were calculated. 


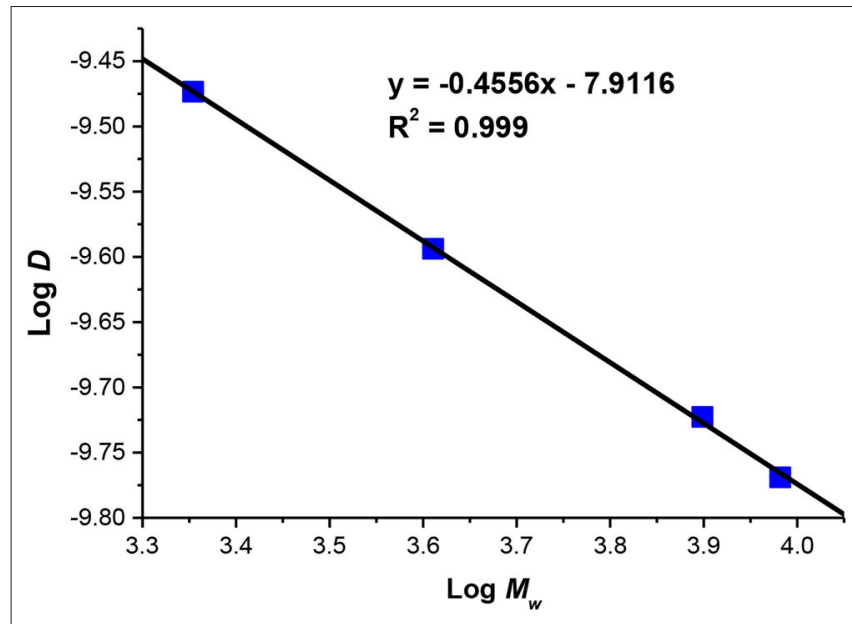

FIGURE 3 | PMMA calibration curve in $\mathrm{CDCl}_{3}$ used for determining $M_{W}$ by DOSY NMR.

\section{Polymerization of MMA With Dark Periods}

In a Schlenk tube, EBPA ( $34 \mu \mathrm{L}, 0.2 \mathrm{mmol}), i$-Pr 2 NEt $(340 \mu \mathrm{L}, 2$ $\mathrm{mmol})$, and Eosin $\mathrm{Y}(2.8 \mathrm{mg}, 4 \mu \mathrm{mol})$ were added to a solution of MMA in DMF ( $8 \mathrm{~mL}, 1: 1 \mathrm{v} / \mathrm{v})$. The reaction mixture was degassed by three freeze-pump-thaw cycles and pumped through the continuous flow photo-microphotoreactor using a syringe pump connected to the flow reactor via a syringe. The reaction mixture was irradiated using Green LEDS for 3 disrupted hours; after each period of $1 \mathrm{~h}$ of irradiation, the solution was kept in the dark under argon for $1 \mathrm{~h}$. For every cycle ( $1 \mathrm{~h}$ "LEDs on," $1 \mathrm{~h}$ "LEDs off"), two samples of $1 \mathrm{~mL}$ each were taken for analysis at the beginning and the end of the "LEDs off" period. During the "LEDs on" period, the reaction mixture was pumped through the flow reactor at a flow rate of $30 \mu \mathrm{L} \cdot \mathrm{min}^{-1}$. Six samples that were taken periodically were precipitated in methanol, filtered, and dried overnight for analysis by GPC and NMR.

\section{Preparation of PMMA-Br Macroinitiator}

In a Schlenk tube, EBPA ( $17 \mu \mathrm{L}, 0.1 \mathrm{mmol}), i-\mathrm{Pr}_{2} \mathrm{NEt}(170 \mu \mathrm{L}, 1$ $\mathrm{mmol})$, and Eosin $\mathrm{Y}(1.4 \mathrm{mg}, 2 \mu \mathrm{mol})$ were added to a solution of MMA in DMF (4 mL, 1:1 v/v). The reaction mixture was degassed by three freeze-pump-thaw cycles and pumped through the continuous flow photo-microphotoreactor using a syringe pump connected to the flow reactor via a syringe. The solution was pumped through the flow system, at a flow rate of $30 \mu \mathrm{L} \cdot \mathrm{min}^{-1}$ (corresponding to $1 \mathrm{~h}$ of residence time) that was irradiated with green LEDs ( $50 \mathrm{~W}, 4500$ Lumens). The polymer was purified by precipitation in methanol. The resulting macroinitiator was dried overnight and characterized by GPC.

\section{Chain Extension of PMMA-Br Macroinitiator With Styrene (St) and Butyl Acrylate (BA)}

PMMA macroinitiator $(0.24 \mathrm{~g}, 0.02 \mathrm{mmol}), i$ - $\operatorname{Pr}_{2} \mathrm{NEt}(35 \mu \mathrm{L}$, $0.2 \mathrm{mmol})$, and Eosin $\mathrm{Y}(0.3 \mathrm{mg}, 0.43 \mu \mathrm{mol})$ were added to a solution of styrene $(450 \mu \mathrm{L}, 4 \mathrm{mmol})$ in $2.5 \mathrm{~mL}$ of DMF

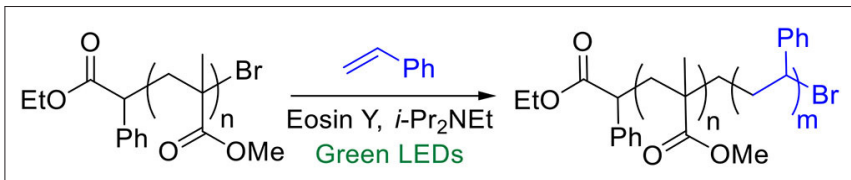

FIGURE 4 | PMMA-Br copolymerization with styrene using Eosin $Y$ in flow.

(Figure 4). The reaction mixture was degassed by three freezepump-thaw cycles and pumped through the continuous flow photo-microphotoreactor using a syringe pump connected to the flow reactor via a syringe. The solution was pumped through the flow system, at a flow rate of $10 \mu \mathrm{L} \cdot \mathrm{min}^{-1}$ (corresponding to $3 \mathrm{~h}$ of residence time) that was irradiated with green LEDs (50 W, 4500 Lumens). The formed copolymer was precipitated, filtered, and dried overnight before its analysis by ${ }^{1} \mathrm{H}$ NMR and GPC.

Similarly, copolymerization with butyl acrylate $(570 \mu \mathrm{L}, 4$ $\mathrm{mmol}$ ) was performed using the same procedure mentioned above. The formed polymer was then filtered, dried, and analyzed by GPC.

\section{RESULTS AND DISCUSSION}

\section{Copper Catalyzed ATRP Using UV Light}

First, we optimized the flow protocol. The homemade continuous flow photo-microreactor was made of FEP tubing $(\varnothing=800 \mu \mathrm{m}$, $\mathrm{L}=1.2 \mathrm{~m}$ ) that can be easily replaced in case of clogging. FEP is transparent to UV so first we performed a classical ATRP in flow catalyzed by $\mathrm{CuBr}_{2}$ under UV irradiation. Compared to the previously described flow experiment, UV-LEDs $(365 \mathrm{~nm})$ with a power of $200 \mathrm{~mW} \cdot \mathrm{cm}^{-2}$ were used instead of a mediumpressure UV lamp, and DMSO was replaced by DMF. We also assessed the ligands PMEDTA and TPMA (Scheme S1) which are commonly used in the literature for Cu-catalyzed ATRP. Best results were obtained with TPMA (Table $\mathbf{S 2}$, entries 1-8) leading to $60 \%$ conversion and low dispersity $(\Theta=1.27)$ compared to the previous results in flow $(\nexists=1.15-1.25)$.

\section{Visible Light ATRP}

We then moved to metallic visible-light photoredox catalysis using the $\mathrm{Ru}(\mathrm{bpy})_{3} \mathrm{Cl}_{2}, i-\mathrm{Pr}_{2} \mathrm{NEt}$ system in DMF irradiated by blue LEDs in flow. The modest dispersity (Table 1 , entry $1 ; D \approx$ 2) obtained is in agreement with the results obtained in batch (Zhang et al., 2011). We then decided to check the activity of Eosin Y, a derivative of fluorescein, which is widely used as visible-light photoredox catalyst for various organic reactions (Van Bergen et al., 1979; Hari et al., 2012; Cantillo et al., 2014; Talla et al., 2015) to perform the polymerization of MMA under flow conditions. Eosin $\mathrm{Y}$ has its maximum absorption at $539 \mathrm{~nm}$ in the visible region of the spectrum and with a high absorption coefficient $\left(\varepsilon=60,800 \mathrm{M}^{-1} \mathrm{~cm}^{-1}\right)$ (Figure S2). The irradiation was afforded by green LEDs with a power of $20 \mathrm{~mW} \cdot \mathrm{cm}^{-2}$.

We originally performed polymerization of MMA in DMF using $\mathrm{EBiB}$ as initiator and Eosin $\mathrm{Y}$ as photocatalyst in both batch (Table 1, entry 2) and flow (Table 1, entry 3). Interestingly, the change from batch to flow system was enough to improve 
TABLE 1 | ATRP polymerization of MMA in DMF in a flow microreactor using LEDs irradiationa .

\begin{tabular}{|c|c|c|c|c|c|c|c|c|c|}
\hline Entry & Catalyst & Initiator/additive & Polymerization conditions ${ }^{a}$ & LEDs & Conv. $(\%)^{\mathrm{b}}$ & Time (min) & $M_{n}$ theoc & $M_{n}{ }^{d}$ & $\nexists^{\mathrm{d}}$ \\
\hline 1 & $\mathrm{Ru}(\mathrm{bpy})_{3} \mathrm{Cl}_{2}$ & $\mathrm{~EB} / \mathrm{B} / \mathrm{i}-\mathrm{Pr}_{2} \mathrm{NEt}$ & 200:1:0.01:10 & Blue & 86 & 430 & 17,200 & 20,600 & 1.96 \\
\hline $2^{e}$ & Eosin $Y$ & $\mathrm{~EB} / \mathrm{B} / \mathrm{i}-\mathrm{Pr}_{2} \mathrm{NEt}$ & 200:1:0.01:10 & Green & 54 & 360 & 11,000 & 24,300 & 1.64 \\
\hline 3 & Eosin $Y$ & $\mathrm{~EB} / \mathrm{B} / \mathrm{i}-\mathrm{Pr}_{2} \mathrm{NEt}$ & 200:1:0.01:10 & Green & 70 & 216 & 14,000 & 25,000 & 1.58 \\
\hline 4 & Eosin $Y$ & $\mathrm{~EB} i \mathrm{~B} / \mathrm{i}-\mathrm{Pr}_{2} \mathrm{NEt}$ & 200:1:0.05:10 & Green & 43 & 216 & 8,600 & 18,000 & 1.86 \\
\hline 5 & Eosin $Y$ & $\mathrm{~EB} / \mathrm{B} / \mathrm{i}-\mathrm{Pr}_{2} \mathrm{Net}$ & 200:1:0.003:10 & Green & 50 & 216 & 10,000 & 17,600 & 1.85 \\
\hline
\end{tabular}

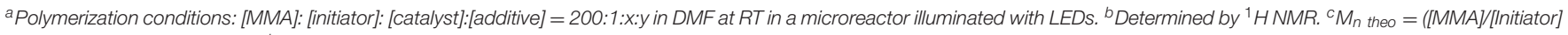
$\times$ conv. $\left.\times M_{M M A}\right)+M_{\text {lnitiator }}{ }^{d}$ Determined by GPC. ${ }^{e}$ Performed in batch.

TABLE 2 | Eosin Y catalyzed ATRP of MMA using EBPA as an initiator in flowa.

\begin{tabular}{lccccc}
\hline Entry & Time (min) & \% Conv. $^{\text {b }}$ & $\boldsymbol{M}_{\boldsymbol{n}}$ theo $^{\mathbf{c}}$ & $\boldsymbol{M}_{\boldsymbol{n}}$ by GPC & $\boldsymbol{\Xi}^{\mathbf{d}}$ \\
\hline 1 & 36 & 20 & 4,280 & 10,400 & 1.46 \\
2 & 45 & 37 & 7,650 & 9,040 & 1.49 \\
3 & 60 & 52 & 10,650 & 12,050 & 1.44 \\
4 & 90 & 63 & 12,860 & 13,050 & 1.36 \\
5 & 120 & 79 & 16,060 & 16,350 & 1.43 \\
6 & 180 & 89 & 18,060 & 18,250 & 1.41 \\
7 & 60 & 53 & 10,650 & 13,000 & 1.42 \\
$8^{\text {f }}$ & 360 & 54 & 11,100 & 24280 & 1.64 \\
$9^{9}$ & 180 & 70 & 14,290 & 19,500 & 1.46 \\
\hline
\end{tabular}

a Polymerization conditions: [MMA]: [EBPA]: [Eosin Y]: [i-Pr2 $N E t]=200: 1: 0.02: 10$ in DMF at RT in microreactor illuminated with green LEDs. ${ }^{b}$ Determined by ${ }^{1} H$ NMR. ${ }^{c} M_{n}$ theo $=\left([M M A) /[E B P A] \times\right.$ conversion $\left.\times M_{M M A}\right)+M_{E B P A}$; where $[M M A]$ and $[E B P A]$ are the concentrations of the monomer and the initiator, respectively and $M_{M M A}$ and $M_{E B P A}$ are their corresponding molar masses. ${ }^{d}$ Determined by GPC. ${ }^{e}$ Used for copolymerization with styrene. ${ }^{f}$ Performed in batch. ${ }^{g}$ Using $p(O M e)$-EBPA synthesized according to the procedure listed by Sharma and Tepe (2005).

remarkably both the rate of the reaction and its control. Although 360 min was required in batch to reach a $50 \%$ conversion, only 216 min was needed in flow for $70 \%$ conversion. Moreover, the dispersity decreased from 1.64 in batch to 1.58 in flow. The results obtained herein in flow are also better than those reported in batch by Yagci's group where 120 min of green LED irradiation of the reaction mixture gave $28 \%$ conversion but with a dispersity of 1.85 (Kutahya et al., 2016).

Following these results, blank experiments were performed where one of the components (catalyst, initiator, $i$ - $\operatorname{Pr}_{2} \mathrm{NEt}$, and light) was removed (Table S3, entry 1). In all of these cases, no PMMA was detected showing that all of these components are essential for the polymerization process. Moreover, since Eosin Y has a high extinction coefficient, a large amount of the catalyst in flow (Table 1, entry 4) will lead to a similar situation to that in batch where the light will only illuminate a small portion of the reactor (Table S1, Figure S3), leading to a decrease in the conversion to $43 \%$. Similarly, decreasing the quantity of Eosin Y will lead to a decrease in the conversion to $50 \%$ despite having a better light penetration in the system as the quantity of the catalyst is not efficient to activate the initiators (Table 1, entry 5). Using EBPA as initiator, which has been previously reported

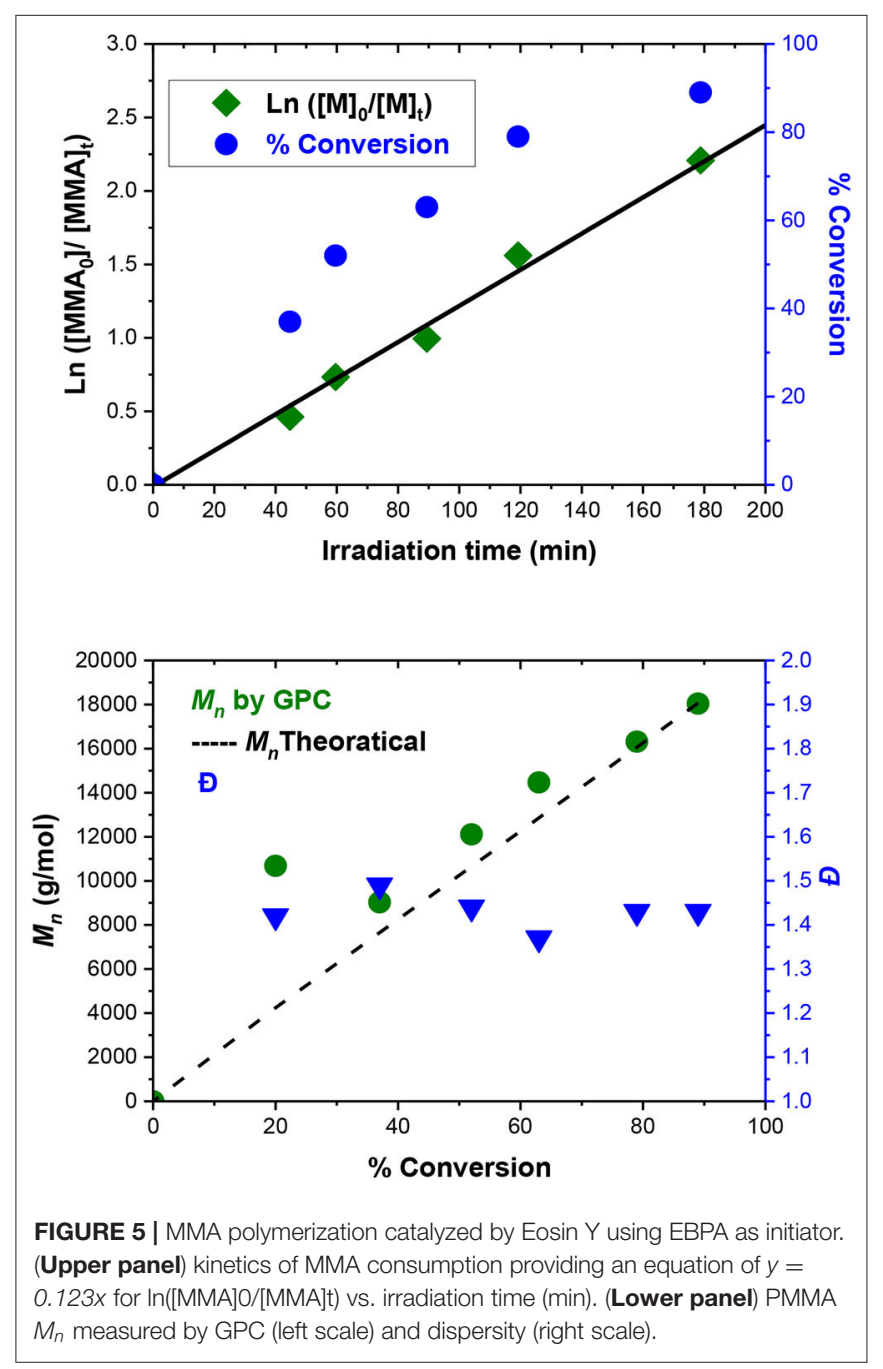

to have a higher activation rate $k_{\text {act }}$ compared to $\mathrm{EBiB}$ due to the radical stability enforced by a phenyl group (Braunecker and Matyjaszewski, 2007), the rate of the polymerization increased remarkably leading to more than $90 \%$ of conversion after only 180 min of irradiation (Table 1, entry 6). The polydispersity was also improved to 1.42 , and the $M_{n}$ values of the polymers formed were closer to the theoretical ones compared to those obtained 

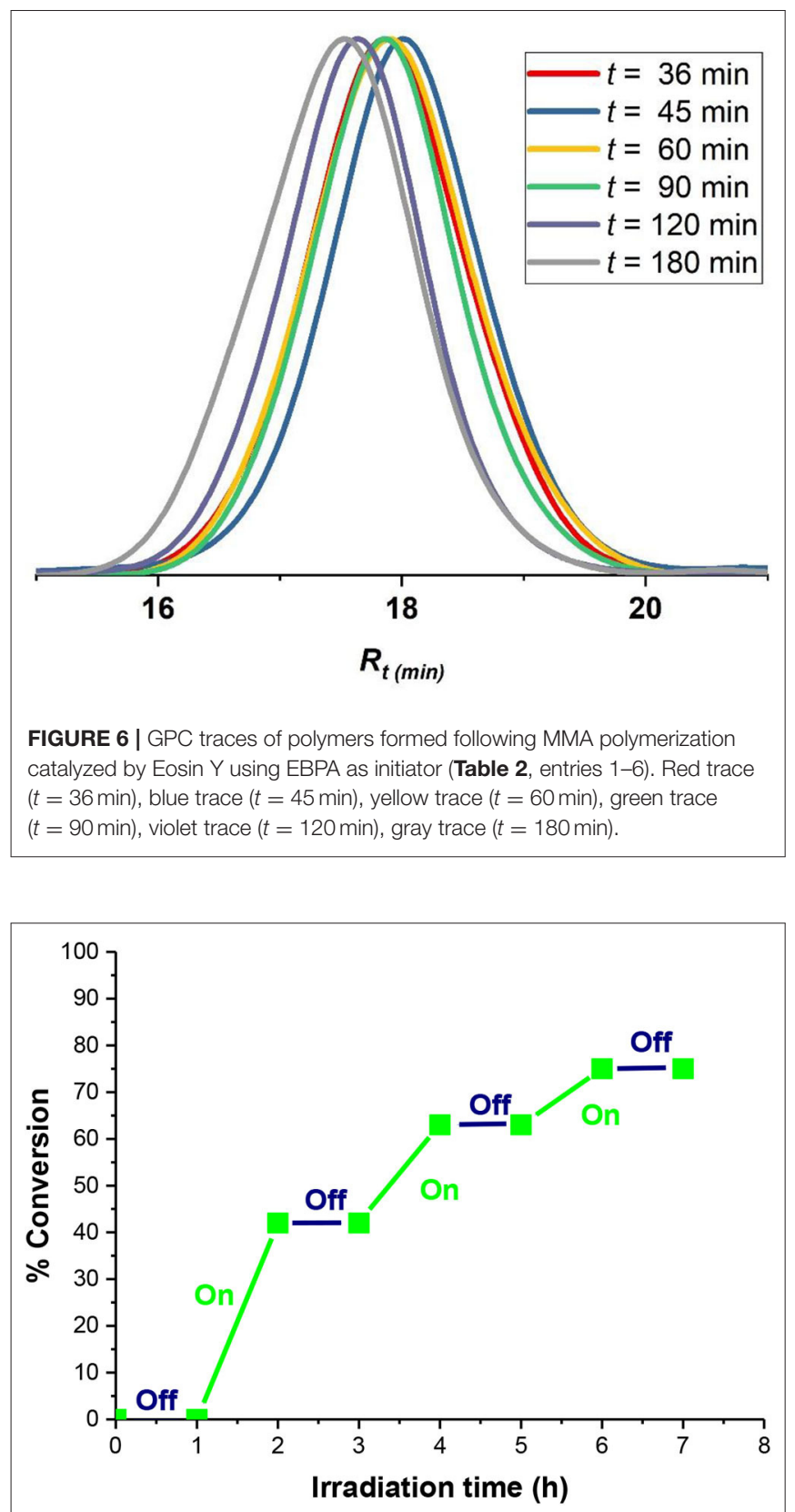

FIGURE 7 | On-off MMA polymerization catalyzed by Eosin Y using EBPA as initiator.

using EBiB (Table S3, Figures S6, S7). To further stabilize the intermediate radical in order to increase the polymerization rate, we used ( $p$-OMe)EBPA as initiator; however, the results were quite disappointing (Table 2, entry 9). The tacticity of the obtained polymers was roughly identical whatever the used ATRP conditions (Table S4, Figure S9).

The best conditions obtained for the ATRP initiated by EBPA (Table 1, entry 6) were further investigated in details (Table 2, entries 1-6). The polymerization follows a first-order kinetics during the whole course of the reaction with an equation

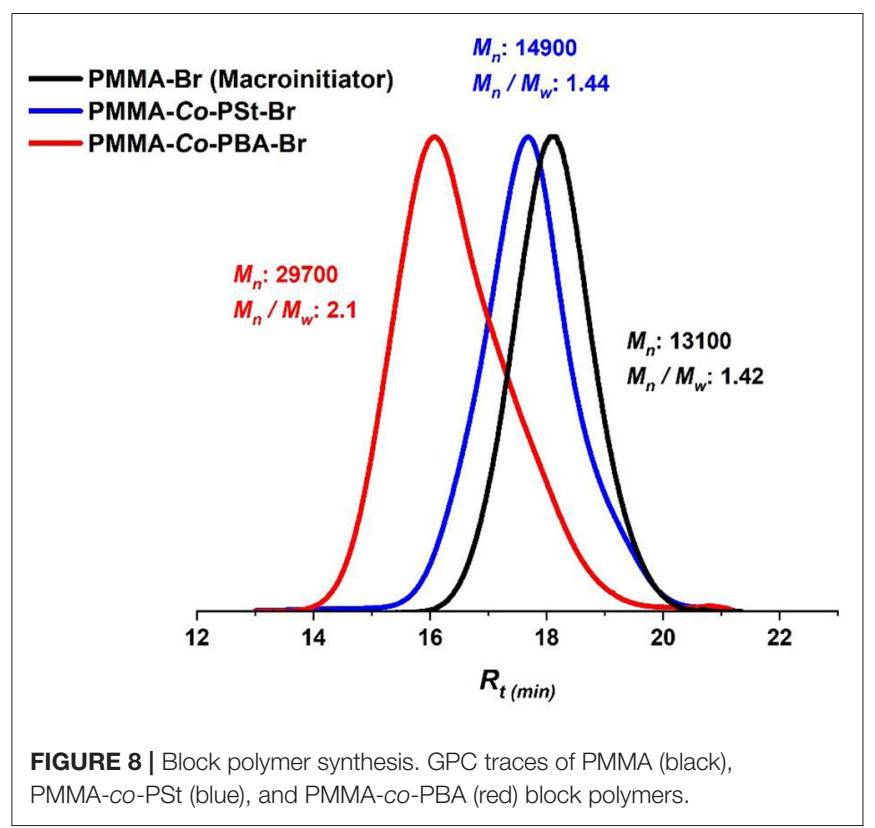

of $y=0.123 x$ for $\ln \left([\mathrm{MMA}]_{0} /[\mathrm{MMA}]_{\mathrm{t}}\right)$ vs. irradiation time (min). The rate constant is $0.123 \mathrm{~min}^{-1}$ (Figure 5, upper panel), suggesting that the concentration of the propagating radicals is almost constant throughout the polymerization. Molecular weights measured by GPC follow the theoretical values starting from $37 \%$ and up to $90 \%$ of conversion suggesting a complete initiation (Figure 5, lower panel; Table 2, entries 1-6). Figure 6, which includes the GPC traces of the polymers of Table 2, entries $1-6$, shows how the increase in the irradiation time leads to the increase in the size of the polymers (GPC traces shifting to lower retention volumes). Moderate values of $Ð$ (1.36-1.49) indicate relatively slow deactivation though still in the range of controlled polymerization $(<1.5)$. Moreover, polymerization in flow gave better conversion and PDI values compared to batch, which highlights the importance of flow settings for photocatalytic ATRP reactions (Table 2 , entry 6 vs. Table 2 entry 9).

However, note that the first point (Table 2, entry 1; Figure 6, red GPC trace) does not follow the theoretical $M_{n}$ so that it deviates upward in Figure 5, Lower panel. This can be attributed to that Eosin $\mathrm{Y}$ undergoes an induction period before it can enter the catalytic cycle (Cornils et al., 2020). As a result, this point was excluded from the kinetic analysis (Figure 5, upper panel).

\section{Livingness of the Formed Polymer}

Controlled "on-off" light switching regulation of polymerization was validated by collecting enough polymer after a given period of irradiation and reinjecting it for the next cycle following a 1-h on/1-h off illumination duration. During the LEDs off periods, the polymerization was paused by having dormant alkyl bromides that were protected from any side radical reaction leading to a stagnant conversion and molecular weight; however, these formed polymers were available for reactivation upon reexposure to light (Figure 7). The Eosin Y-photoinduced ATRP in flow ceased when the light was turned off and started again 


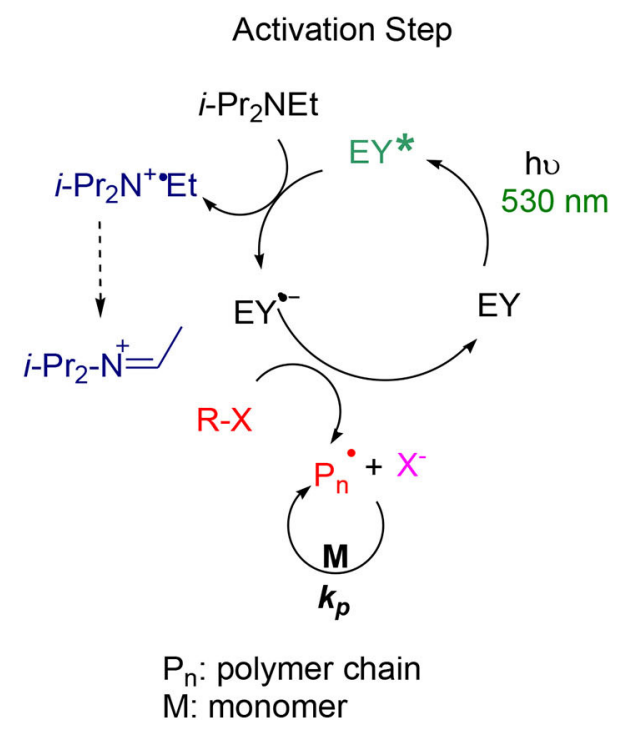

Reductive propagation step
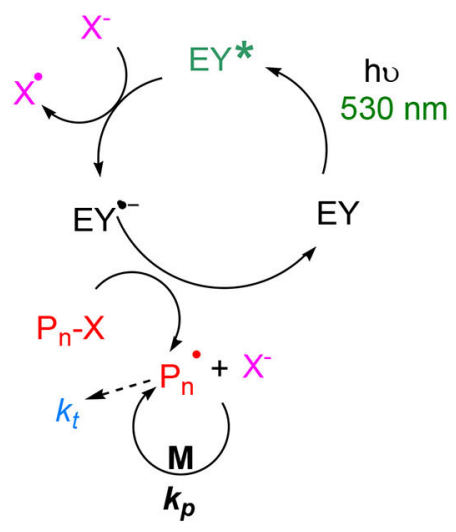

$k_{p}$ : propagation rate constant

$k_{t}$ : termination rate constant

FIGURE 9 | Suggested photoinduced electron transfer ATRP mechanism by the Eosin $\mathrm{Y} / \mathrm{i}-\mathrm{Pr}_{2} \mathrm{NEt}$ catalyst.

TABLE $3 \mid M_{w}$ of PMMA synthesized in flow by DOSY NMR and GPC.

\begin{tabular}{lcccc}
\hline Entry & $\begin{array}{c}\boldsymbol{D} \times \mathbf{1 0}^{-\mathbf{1 0}} \text { by } \\
\text { DosY }\left(\mathbf{m}^{\mathbf{2}} \mathbf{. s}^{-\mathbf{1}}\right)\end{array}$ & $\boldsymbol{M}_{\boldsymbol{w}}$ by DosYa & $\boldsymbol{M}_{\boldsymbol{w}}$ by GPC. & $\%$ diff $^{\mathbf{b}}$ \\
\hline 1 & 1.10 & 24,920 & 24,900 & 0.08 \\
2 & 1.20 & 20,740 & 20,270 & 2.32 \\
3 & 1.25 & 19,100 & 19,150 & -0.26 \\
4 & 1.26 & 18,710 & 18,040 & 3.70 \\
5 & 1.28 & 18,020 & 17,980 & 0.22 \\
6 & 1.30 & 17,550 & 17,290 & 1.50 \\
7 & 1.96 & 7,190 & 6,810 & 5.58 \\
\hline
\end{tabular}

${ }^{a}$ Equation of the PMMA standard calibration curve is $\log D=-0.4656 \log M_{w}-7.9116$ was used to determine $M_{w}$ using the values of $D$ provided by DOSY NMR. ${ }^{b}$ Calculated from $M_{W}(G P C)$ and $M_{w}(D O S Y)$.

in response to the irradiation. Therefore, the control over the formation and termination of active species can be performed by using a simple on-off operation of light during Eosin Yphotoinduced ATRP in flow leading to $M_{n}$ (experimental) $\sim \mathrm{M}_{n}$ (theoretical).

The "livingness" of the Eosin Y-photoinduced ATRP and the termination of the formed polymers by an active bromide ion were further demonstrated by a copolymerization reaction of styrene with a PMMA-Br macroinitiator (Figure 4). The PMMA-Br macroinitiator was firstly synthesized by photoinduced ATRP in flow to get PMMA-Br (Table 2, entry 7, $M_{n}=13,100, Ð=1.42$ ) and then used as a macroinitiator for Eosin Y-catalyzed ATRP of styrene (St) and butyl acrylate (BA). The GPC traces of the macroinitiator and the corresponding copolymers are displayed in Figure 8, and the NMR spectrum of PMMA-co-PSt is represented in Figure S8. The results of PMMA-co-PBA (Figure 8, red trace) were not very convenient as the PDI value was 2.1, which signifies a loss in control, which can be attributed with the high reactivity of butyl acrylate that requires strictly anhydrous and oxygen-free conditions (Roos et al., 1999). However, the clear shift of $M_{n}$ of PMMA-co-PSt to a higher molecular weight while still having a good PDI (Figure 8, blue trace) and the presence of peaks that correspond to PSt and PMMA in the NMR spectrum indicate an effective copolymerization by reinitiation. These results of PSt copolymerization are in accordance with the literature where clear shifts to lower retention volumes were also reported by performing PMMA chain extension and copolymerization with PSt using the same condition but in batch (Kutahya et al., 2016; Yilmaz and Yagci, 2018).

\section{Mechanism of Eosin Y-Catalyzed Photoinduced ATRP}

The suggested mechanism of the Eosin Y-photoinduced electron transfer (PET)-ATRP is represented in Figure 9. All the standard potentials are in ACN vs. SCE (Wayner et al., 1986; Lin et al., 2008; Isse et al., 2010; Hari and König, 2014; Pan et al., 2016a; Romero and Nicewicz, 2016). Upon irradiation with green LEDs, Eosin $\mathrm{Y}$ affords the excited state $\mathrm{EY}^{*}$ which has a high oxidation potential $\left(E^{0}\left(\mathrm{EY} / \mathrm{EY}^{*}\right)=1.89 \mathrm{~V}\right)$ (Hari and König, 2014). In the activation step, the electron donor, $i$ - $\operatorname{Pr}_{2} \mathrm{NEt}$, reductively quenches $\mathrm{EY}^{*}$ by a single electron transfer to form a radical anion $\mathrm{EY}^{\bullet}$ - and an amine radical cation $i-\mathrm{Pr}_{2} \mathrm{~N}^{\bullet}+$ Et intermediate $\left(E^{\circ}\left(i-\operatorname{Pr}_{2} \mathrm{NEt} / i-\operatorname{Pr}_{2} \mathrm{~N}^{\bullet}+\mathrm{Et}\right) \approx 1.0 \mathrm{~V}\right)$. The latter rearranges almost at a diffusion rate to the $C$-centered radical $i$-PrEtNC ${ }^{\bullet}\left(\mathrm{CH}_{3}\right)_{2}$ or $i-\mathrm{Pr}_{2} \mathrm{NCH}^{\bullet} \mathrm{CH}_{3}$ (Wayner et al., 1986; Romero and Nicewicz, 2016). The radical anion $\mathrm{EY}^{\bullet}-\left(E^{0}(\mathrm{EY} \bullet-/ \mathrm{EY})=-1.06 \mathrm{~V}\right)$, or the reductive $C$-centered radical $\left(E_{0}(C\right.$-centered radical/iminium $)=$ $-1.12 \mathrm{~V})$, then transfers an electron to the alkyl bromide $\mathrm{EB} i \mathrm{~B}$ or EBPA $\left(E^{0}\left(\mathrm{RX} / \mathrm{R}^{\bullet}+\mathrm{Br}^{-}\right)=-0.42 \mathrm{~V}\right.$ and $-0.20 \mathrm{~V}$, respectively) (Lin et al., 2008). The $\mathrm{EB} i \mathrm{~B}$ or EBPA radical anion cleaves generating an alkyl radical that adds to the monomer-inducing 
propagation. In the reductive propagation step, the excited Eosin $\mathrm{Y}$ recaptures an electron by oxidizing either the bromide ion $\mathrm{Br}^{-}$into bromine radical $\mathrm{Br}^{\bullet}\left(E^{0}\left(\mathrm{Br}^{-} / \mathrm{Br}^{\bullet}\right)=1.75 \mathrm{~V}\right.$ ) (Isse et al., 2010), or the complex propagating radical-bromide ion by a concerted electron transfer (Pan et al., 2016a). The bromine radical $\mathrm{Br}^{\bullet}$ deactivates propagation and forms the dormant polymer. The formed radical anion $\mathrm{EY}^{\bullet}-$ is reduced to back to EY by providing an electron to the dormant polymer, which has the same structure as a tertiary $\alpha$-bromoester that reacts with the monomer. The consumption of the sacrificial amine is to compensate the irreversible terminations of the propagating cycle.

In this mechanism, the very unlikely oxidation of $\mathrm{Br}^{-}$by the radical cation $i-\mathrm{Pr}_{2} \mathrm{~N}^{\bullet}+$ Et (Liu et al., 2016) or the chain radical oxidation to a cation (Xu et al., 2014) is avoided; only a catalytic amount of the sacrificial amine is required to initiate the reaction. Furthermore, all of the steps have favorable redox potentials. This mechanism shows that Eosin Y has well-established activation deactivation processes that result in the control of the molecular weights and dispersities of the formed polymers.

\section{DOSY vs. GPC}

Based on the calibration curve obtained using DOSY NMR (Figure 3), $M_{w}$ values of selected polymers were obtained and are presented in Table 3 along with the corresponding $M_{w}$ values obtained by GPC. Interestingly, the two methods almost gave the same $M_{w}$ values in all of the entries. The \% of difference between both values varies between $0.08 \%$ in entry 1 and a maximum value of only $5.5 \%$ in entry 7 . This shows that DOSY NMR can be a good analytical method for the characterization of polymers. NMR spectroscopy can provide the full characterization of the polymer: \% conversion, tacticity, $M_{n}$ by ${ }^{1} \mathrm{H}$ NMR, and $M_{w}$ by DOSY NMR, rendering it complementary to GPC.

\section{CONCLUSION}

In conclusion, we have demonstrated that ATRP of MMA using Eosin $\mathrm{Y}$ as a photocatalyst in a flow reactor illuminated by green LEDs is very efficient, affording $90 \%$ of conversion in $3 \mathrm{~h}$. Perfect first-order kinetics, full-initiation or dormant polymer activation, moderate dispersity, and masses in agreement with the theoretical values were obtained showing the great mechanistic and synthetic potentials of our conditions. The main reason for this improvement is the homogeneous illumination in flow

\section{REFERENCES}

Alfredo, N. V., Jalapa, N. E., Morales, S. L., Ryabov, A. D., Le Lagadec, R., and Alexandrov, L. (2012). Light-driven living/controlled radical polymerization of hydrophobic monomers catalyzed by ruthenium(II) metalacycles. Macromolecules 45, 8135-8146. doi: 10.1021/ma30 14383

Anastasaki, A., Nikolaou, V., Zhang, Q., Burns, J., Samanta, S. R., Waldron, C., et al. (2014). Copper(II)/tertiary amine synergy in photoinduced living radical polymerization: accelerated synthesis of omega-functional and alpha,omega-heterofunctional poly(acrylates). J. Am. Chem. Soc. 136, 1141-1149. doi: $10.1021 / \mathrm{ja} 411780 \mathrm{~m}$ reactors and hence the shorter reaction time compared to batch (Cambié et al., 2016). We also have shown that NMR spectroscopy (1D and 2D) could be considered as a reliable tool for characterization of polymers. This work combines three essential components toward a "greener" chemistry: miniaturization, renewable energy, and metal-free catalysis that can be used later for scaling up. This work is the first in a series of reactions that our lab is assessing that includes extending the current continuous-flow system to further improve the irradiation efficiency, synthesizing more complex polymers and using this system for other types of RDRPs.

\section{DATA AVAILABILITY STATEMENT}

The original contributions presented in the study are included in the article/Supplementary Material, further inquiries can be directed to the corresponding author/s.

\section{AUTHOR CONTRIBUTIONS}

All authors listed have made a substantial, direct and intellectual contribution to the work, and approved it for publication.

\section{FUNDING}

This work was partially funded by LASeR Association-Lebanon in a form of Ph.D. Scholarship to NE. The NMR and mass spectrometry facilities used in this study were funded by the European Community (ERDF), Région Haut de France (France), the CNRS, and the Université de Lille, Faculté de Sciences et Technologies.

\section{ACKNOWLEDGMENTS}

We would like to thank Dr. Benjamin Nottelet, Dr. Vincent Darcosand, the team at the Institut des Biomolécules Max Mousseron (IBMM) at Université de Monpellier for providing access to the GPC at their facilities.

\section{SUPPLEMENTARY MATERIAL}

The Supplementary Material for this article can be found online at: https://www.frontiersin.org/articles/10.3389/fchem. 2020.00740/full\#supplementary-material 
Buss, B. L., and Miyake, G. M. (2018). Photoinduced controlled radical polymerizations performed in flow: methods, products, and opportunities. Chem. Mater. 30, 3931-3942. doi: 10.1021/acs.chemmater.8b01359

Cambié, D., Bottecchia, C., Straathof, N. J., Hessel, V., and Noël, T. (2016). Applications of continuous-flow photochemistry in organic synthesis, material science, and water treatment. Chem. Rev. 116, 10276-10341. doi: 10.1021/acs.chemrev.5b00707

Cantillo, D., De Frutos, O., Rincó, N. J. A., Mateos, C., and Kappe, C. O. (2014). Continuous flow $\alpha$-trifluoromethylation of ketones by metal-free visible light photoredox catalysis. Org. Lett. 16, 896-899. doi: 10.1021/ol403650y

Chatani, S., Kloxin, C. J., and Bowman, C. N. (2014). The power of light in polymer science: photochemical processes to manipulate polymer formation, structure, and properties. Polym. Chem. 5, 2187-2201. doi: 10.1039/C3PY01334K

Chen, M., Zhong, M., and Johnson, J. A. (2016). Light-controlled radical polymerization: mechanisms, methods, and applications. Chem. Rev. 116, 10167-10211. doi: 10.1021/acs.chemrev.5b00671

Chmielarz, P., Fantin, M., Park, S., Isse, A. A., Gennaro, A., Magenau, A. J., et al. (2017). Electrochemically mediated atom transfer radical polymerization (eATRP). Prog. Polym. Sci. 69, 47-78. doi: 10.1016/j.progpolymsci.2017. 02.005

Cornils, B., Herrmann, W. A., Xu, J.-H., and Zanthoff, H.-W. (2020). Catalysis from A to Z: a concise encyclopedia. Weinheim: John Wiley and Sons. doi: 10.1002/9783527809080

Corrigan, N., Almasri, A., Taillades, W., Xu, J., and Boyer, C. (2017). Controlling molecular weight distributions through photoinduced flow polymerization. Macromolecules 50, 8438-8448. doi: 10.1021/acs.macromol.7b01890

Corrigan, N., Yeow, J., Judzewitsch, P., Xu, J., and Boyer, C. (2019). Seeing the light: advancing materials chemistry through photopolymerization. Angew. Chem. Int. Ed. Engl. 58, 5170-5189. doi: 10.1002/anie.201805473

Discekici, E. H., Anastasaki, A., Read De Alaniz, J., and Hawker, C. J. (2018). Evolution and future directions of metal-free atom transfer radical polymerization. Macromolecules 51, 7421-7434. doi: 10.1021/acs.macromol.8b01401

Elliott, L. D., Knowles, J. P., Koovits, P. J., Maskill, K. G., Ralph, M. J., Lejeune, G., et al. (2014). Batch versus flow photochemistry: a revealing comparison of yield and productivity. Chem. Eur. J. 20, 15226-15232. doi: 10.1002/chem. 201404347

Fantin, M., Chmielarz, P., Wang, Y., Lorandi, F., Isse, A. A., Gennaro, A., et al. (2017). Harnessing the interaction between surfactant and hydrophilic catalyst to control e ATRP in miniemulsion. Macromolecules 50, 3726-3732. doi: 10.1021/acs.macromol.7b00530

Fors, B. P., and Hawker, C. J. (2012). Control of a living radical polymerization of methacrylates by light. Angew. Chem. Int. Ed. Engl. 51, 8850-8853. doi: 10.1002/anie.201203639

Gao, H., and Matyjaszewski, K. (2006). Synthesis of star polymers by a combination of ATRP and the "click" coupling method. Macromolecules 39, 4960-4965. doi: $10.1021 / \mathrm{ma} 060926 \mathrm{c}$

Garlets, Z. J., Nguyen, J. D., and Stephenson, C. R. J. (2014). The development of visible-light photoredox catalysis in flow. Isr. J. Chem. 54, 351-360. doi: $10.1002 /$ ijch.201300136

Hari, D. P., and König, B. (2014). Synthetic applications of eosin Y in photoredox catalysis. Chem. Commun. 50, 6688-6699. doi: 10.1039/C4CC00751D

Hari, D. P., Schroll, P., and König, B. (2012). Metal-free, visible-light-mediated direct $\mathrm{C}-\mathrm{H}$ arylation of heteroarenes with aryl diazonium salts. J. Am. Chem. Soc. 134, 2958-2961. doi: 10.1021/ja212099r

Hu, X., Zhang, Y., Cui, G., Zhu, N., and Guo, K. (2017). Poly (vinylidene fluoride-co-chlorotrifluoroethylene) modification via organocatalyzed atom transfer radical polymerization. Macromol. Rapid Commun. 38:1700399. doi: 10.1002/marc.201700399

Hu, X., Zhu, N., Fang, Z., Li, Z., and Guo, K. (2016). Continuous flow coppermediated reversible deactivation radical polymerizations. Eur. Polym. J. 80, 177-185. doi: 10.1016/j.eurpolymj.2016.04.006

Isse, A. A., Lin, C. Y., Coote, M. L., and Gennaro, A. (2010). Estimation of standard reduction potentials of halogen atoms and alkyl halides. J. Phys. Chem. B. 115, 678-684. doi: 10.1021/jp109613t

Konkolewicz, D., Schroder, K., Buback, J., Bernhard, S., and Matyjaszewski, K. (2012). Visible light and sunlight photoinduced ATRP with ppm of Cu catalyst. ACS Macro Lett. 1, 1219-1223. doi: 10.1021/mz300457e
Kutahya, C., Aykac, F. S., Yilmaz, G., and Yagci, Y. (2016). LED and visible lightinduced metal free ATRP using reducible dyes in the presence of amines. Polym. Chem. 7, 6094-6098. doi: 10.1039/C6PY01417H

Lin, C. Y., Coote, M. L., Gennaro, A., and Matyjaszewski, K. (2008). Ab initio evaluation of the thermodynamic and electrochemical properties of alkyl halides and radicals and their mechanistic implications for atom transfer radical polymerization. J. Am. Chem. Soc. 130, 12762-12774. doi: 10.1021/ja8038823

Liu, X., Zhang, L., Cheng, Z., and Zhu, X. (2016). Metal-free photoinduced electron transfer-atom transfer radical polymerization (PET-ATRP) via a visible light organic photocatalyst. Polym. Chem. 7, 689-700. doi: 10.1039/C5PY01765C

Ma, W. C., Chen, D., Wang, L., Ma, Y. H., Zhao, C. W., and Yang, W. T. (2015). Visible light-controlled radical polymerization of propargyl methacrylate activated by a photoredox catalyst fac- Ir(ppy)(3). J. Macromol. Sci. Pure Appl. Chem. 52, 761-769. doi: 10.1080/10601325.2015.1063883

Ma, W. C., Chen, H. C., Ma, Y. H., Zhao, C. W., and Yang, W. T. (2014). Visible-light-induced controlled polymerization of hydrophilic monomers with $\operatorname{Ir}($ ppy)(3) as a photoredox catalyst in anisole. Macromol. Chem. Phys. 215, 1012-1021. doi: 10.1002/macp.201400024

Matyjaszewski, K. (2012). Atom Transfer Radical Polymerization (ATRP): current status and future perspectives. Macromolecules 45, 4015-4039. doi: $10.1021 / \mathrm{ma} 3001719$

Matyjaszewski, K., and Tsarevsky, N. V. (2009). Nanostructured functional materials prepared by atom transfer radical polymerization. Nat. Chem. 1, 276-288. doi: $10.1038 /$ nchem.257

Matyjaszewski, K., and Tsarevsky, N. V. (2014). Macromolecular engineering by atom transfer radical polymerization. J. Am. Chem. Soc. 136, 6513-6533. doi: $10.1021 /$ ja408069v

Matyjaszewski, K., and Xia, J. H. (2001). Atom transfer radical polymerization. Chem. Rev. 101, 2921-2990. doi: 10.1021/cr940534g

Melker, A., Fors, B. P., Hawker, C. J., and Poelma, J. E. (2015). Continuous flow synthesis of poly(methyl methacrylate) via a light-mediated controlled radical polymerization. J. Polym. Sci. A. Polym. Chem. 53, 2693-2698. doi: $10.1002 /$ pola.27765

Miyake, G. M., and Theriot, J. C. (2014). Perylene as an organic photocatalyst for the radical polymerization of functionalized vinyl monomers through oxidative quenching with alkyl bromides and visible light. Macromolecules 47, 8255-8261. doi: 10.1021/ma502044f

Mosnacek, J., and Ilcikova, M. (2012). Photochemically mediated atom transfer radical polymerization of methyl methacrylate using ppm amounts of catalyst. Macromolecules 45, 5859-5865. doi: 10.1021/ma300773t

Myers, R. M., Fitzpatrick, D. E., Turner, R. M., and Ley, S. V. (2014). Flow chemistry meets advanced functional materials. Chem. Eur. J. 20, 12348-12366. doi: 10.1002/chem.201402801

Ouchi, M., Terashima, T., and Sawamoto, M. (2008). Precision control of radical polymerization via transition metal catalysis: from dormant species to designed catalysts for precision functional polymers. Acc. Chem. Res. 41, 1120-1132. doi: 10.1021/ar800063t

Ouchi, M., Terashima, T., and Sawamoto, M. (2009). Transition metal-catalyzed living radical polymerization: toward perfection in catalysis and precision polymer synthesis. Chem. Rev. 109, 4963-5050. doi: 10.1021/cr900234b

Pan, X., Fang, C., Fantin, M., Malhotra, N., So, W. Y., Peteanu, L. A., et al. (2016a). Mechanism of photoinduced metal-free atom transfer radical polymerization: experimental and computational studies. J. Am. Chem. Soc. 138, 2411-2425. doi: $10.1021 /$ jacs. 5 b 13455

Pan, X., Tasdelen, M. A., Laun, J., Junkers, T., Yagci, Y., and Matyjaszewski, K. (2016b). Photomediated controlled radical polymerization. Prog. Polym. Sci. 62, 73-125. doi: 10.1016/j.progpolymsci.2016.06.005

Pan, X. C., Lamson, M., Yan, J. J., and Matyjaszewski, K. (2015). Photoinduced metal-free atom transfer radical polymerization of acrylonitrile. ACS Macro Lett. 4, 192-196. doi: 10.1021/mz500834g

Priyadarshani, N., Liang, Y. N., Suriboot, J., Bazzi, H. S., and Bergbreiter, D. E. (2013). Recoverable reusable Polyisobutylene (PIB)-Bound Ruthenium Bipyridine (Ru(PIB-bpy)(3)Cl-2) photoredox polymerization catalysts. ACS Macro Lett. 2, 571-574. doi: 10.1021/mz400232y

Ramakers, G., Krivcov, A., Trouillet, V., Welle, A., Möbius, H., and Junkers, T. (2017). Organocatalyzed photo-atom transfer radical polymerization of methacrylic acid in continuous flow and surface grafting. Macromol. Rapid Commun. 38:1700423. doi: 10.1002/marc.201700423 
Ramsey, B. L., Pearson, R. M., Beck, L. R., and Miyake, G. M. (2017). Photoinduced organocatalyzed atom transfer radical polymerization using continuous flow. Macromolecules 50, 2668-2674. doi: 10.1021/acs.macromol.6b02791

Reis, M. H., Leibfarth, F. A., and Pitet, L. M. (2020). Polymerizations in continuous flow: recent advances in the synthesis of diverse polymeric materials. ACS Macro Lett. 9, 123-133. doi: 10.1021/acsmacrolett.9b00933

Ribelli, T. G., Konkolewicz, D., Bernhard, S., and Matyjaszewski, K. (2014). How are radicals (Re)generated in photochemical ATRP? J. Am. Chem. Soc. 136, 13303-13312. doi: 10.1021/ja506379s

Ribelli, T. G., Lorandi, F., Fantin, M., and Matyjaszewski, K. (2019). Atom transfer radical polymerization: billion times more active catalysts and new initiation systems. Macromol. Rapid Commun. 40:1800616. doi: 10.1002/marc. 201800616

Romero, N. A., and Nicewicz, D. A. (2016). Organic photoredox catalysis. Chem. Rev. 116, 10075-10166. doi: 10.1021/acs.chemrev.6b00057

Roos, S. G., Müller, A. H., and Matyjaszewski, K. (1999). Copolymerization of n-butyl acrylate with methyl methacrylate and PMMA macromonomers: comparison of reactivity ratios in conventional and atom transfer radical copolymerization. Macromolecules 32, 8331-8335. doi: 10.1021/ma 9819337

Rubens, M., Latsrisaeng, P., and Junkers, T. (2017). Visible light-induced iniferter polymerization of methacrylates enhanced by continuous flow. Polym. Chem. 8, 6496-6505. doi: 10.1039/C7PY01157A

Ryan, M. D., Pearson, R. M., French, T. A., and Miyake, G. M. (2017). Impact of light intensity on control in photoinduced organocatalyzed atom transfer radical polymerization. Macromolecules 50, 4616-4622. doi: 10.1021/acs.macromol.7b00502

Sharma, V., and Tepe, J. J. (2005). Diastereochemical diversity of imidazoline scaffolds via substrate controlled TMSCl mediated cycloaddition of azlactones. Org. Lett. 7, 5091-5094. doi: 10.1021/ol052118w

Su, Y. H., Straathof, N. J. W., Hessel, V., and Noel, T. (2014). Photochemical transformations accelerated in continuous-flow reactors: basic concepts and applications. Chem. Eur. J. 20, 10562-10589. doi: 10.1002/chem.201400283

Talla, A., Driessen, B., Straathof, N. J., Milroy, L. G., Brunsveld, L., Hessel, V., et al. (2015). Metal-free photocatalytic aerobic oxidation of thiols to disulfides in batch and continuous-flow. Adv. Synth. Catal. 357, 2180-2186. doi: 10.1002/adsc.201401010

Tasdelen, M. A., Uygun, M., and Yagci, Y. (2011). Photoinduced controlled radical polymerization. Macromol. Rapid Commun. 32, 58-62. doi: 10.1002/marc.201000351

Theriot, J. C., Lim, C. H., Yang, H., Ryan, M. D., Musgrave, C. B., and Miyake, G. M. (2016). Organocatalyzed atom transfer radical polymerization driven by visible light. Science 352, 1082-1086. doi: 10.1126/science.aaf3935

Tonhauser, C., Nataello, A., Lowe, H., and Frey, H. (2012). Microflow technology in polymer synthesis. Macromolecules 45, 9551-9570. doi: 10.1021/ma301671x
Treat, N. J., Fors, B. P., Kramer, J. W., Christianson, M., Chiu, C. Y., De Alaniz, J. R., et al. (2014a). Controlled radical polymerization of acrylates regulated by visible light. ACS Macro Lett. 3, 580-584. doi: 10.1021/mz500242a

Treat, N. J., Sprafke, H., Kramer, J. W., Clark, P. G., Barton, B. E., Read De Alaniz, J., et al. (2014b). Metal-free atom transfer radical polymerization. J. Am. Chem. Soc. 136, 16096-16101. doi: 10.1021/ja510389m

Van Bergen, T., Hedstrand, D. M., Kruizinga, W. H., and Kellogg, R. M. (1979). Chemistry of dihydropyridines. 9. Hydride transfer from 1, 4-dihydropyridines to sp3-hybridized carbon in sulfonium salts and activated halides. Studies with NAD (P) H models. J. Org. Chem. 44, 4953-4962. doi: 10.1021/jo00394a044

Wayner, D. D., Dannenberg, J. J., and Griller, D. (1986). Oxidation potentials of $\alpha$-aminoalkyl radicals: bond dissociation energies for related radical cations. Chem. Phys. Lett. 131, 189-191. doi: 10.1016/0009-2614(86)80542-5

Wenn, B., Conradi, M., Carreiras, A. D., Haddleton, D. M., and Junkers, T. (2014). Photo-induced copper-mediated polymerization of methyl acrylate in continuous flow reactors. Polym. Chem. 5, 3053-3060. doi: 10.1039/C3PY01762A

Xu, J. T., Atme, A., Martins, A. F. M., Jung, K., and Boyer, C. (2014). Photoredox catalyst-mediated atom transfer radical addition for polymer functionalization under visible light. Polym. Chem. 5, 3321-3325. doi: 10.1039/C4PY00193A

Yang, Q. Z., Dumur, F., Morlet-Savary, F., Poly, J., and Lalevee, J. (2015). Photocatalyzed Cu-based ATRP involving an oxidative quenching mechanism under visible light. Macromolecules 48, 1972-1980. doi: 10.1021/ma502384y

Yilmaz, G., and Yagci, Y. (2018). Photoinduced metal-free atom transfer radical polymerizations: state-of-the-art, mechanistic aspects and applications. Polym. Chem. 9, 1757-1762. doi: 10.1039/C8PY00207J

Zhang, C., Wang, L., Jia, D., Yan, J., and Li, H. (2019). Microfluidically mediated atom-transfer radical polymerization. Chem. Commun. 55, 7554-7557. doi: 10.1039/C9CC04061G

Zhang, G., Song, I. Y., Ahn, K. H., Park, T., and Choi, W. (2011). Free radical polymerization initiated and controlled by visible light photocatalysis at ambient temperature. Macromolecules 44, 7594-7599. doi: 10.1021/ma201546c

Conflict of Interest: The authors declare that the research was conducted in the absence of any commercial or financial relationships that could be construed as a potential conflict of interest.

Copyright (C) 2020 El Achi, Bakkour, Adhami, Molina, Penhoat, Azaroual, ChaussetBoissarie and Rolando. This is an open-access article distributed under the terms of the Creative Commons Attribution License (CC BY). The use, distribution or reproduction in other forums is permitted, provided the original author(s) and the copyright owner(s) are credited and that the original publication in this journal is cited, in accordance with accepted academic practice. No use, distribution or reproduction is permitted which does not comply with these terms. 